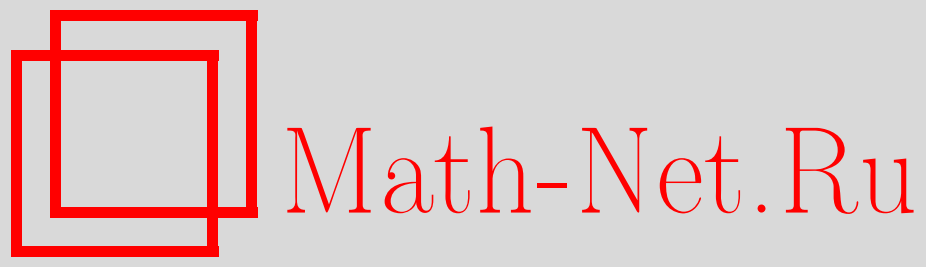

В. Э. Адлер, А. И. Бобенко, Ю. Б. Сурис, Дискретные нелинейные гиперболические уравнения. Классификация интегрируемых случаев, Функи. анализ и его прил., 2009, том 43, выпуск 1, 3-21

DOI: https://doi.org/10.4213/faa2936

Использование Общероссийского математического портала MathNet.Ru подразумевает, что вы прочитали и согласны с пользовательским соглашением

http://www.mathnet.ru/rus/agreement

Параметры загрузки:

IP : 54.198 .55 .26

26 апреля 2023 г., 11:58:44

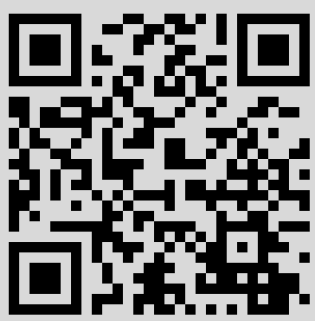




\title{
Дискретные нелинейные гиперболические уравнения. Классификация интегрируемых случаев*
}

\author{
(с) 2009. В. Э. АДлеР, А. И. БоБЕнко, Ю. Б. СуриС
}

\begin{abstract}
Мы рассматриваем дискретные нелинейные гиперболические уравнения на квадграфах, в частности на решетке $\mathbb{Z}^{2}$. Поля ассоциированы с вершинами, и уравнение $Q\left(x_{1}, x_{2}, x_{3}, x_{4}\right)=0$ связывает четыре вершины одной ячейки. Интегрируемость уравнений понимается как 3D-совместность. Это означает, что уравнения одного и того же типа можно приписать всем граням трехмерного куба так, что полученная система будет совместной. Это позволяет также распространить данные уравнения на многомерные решетки $\mathbb{Z}^{N}$. Мы классифицируем интегрируемые уравнения с комплексными полями $x$ и с $Q$, являющимся мультиаффинным многочленом по всем аргументам. Метод основан на анализе сингулярных решений.
\end{abstract}

\section{$\S 1$. Введение}

Концепция совместности является центральной в теории интегрируемых систем. Она появляется уже в самом определении вполне интегрируемого по Лиувиллю-Арнольду гамильтонова потока, которое гласит, что поток должен быть включен в полное семейство коммутирующих (совместных) гамильтоновых потоков [1]. Аналогично, для солитонных (интегрируемых) уравнений в частных производных характерно то, что они не являются изолированными объектами, но всегда организованы в иерархии совместных систем. Условие существования набора коммутирующих систем может быть положено в основу симметрийного подхода в задаче о выделении и классификации интегрируемых случаев среди того или иного типа уравнений общего вида [18]. Еще одним проявлением идеи совместности является связь между непрерывными и дискретными системами, основанная на понятии преобразования Бэклунда и теореме Бъянки о перестановочности [9]. Последняя играет роль одного из фундаментальных принципов в дискретной дифференциальной геометрии [12].

Итак, совместность дискретных уравнений выходит на центральную сцену. Мы говорим, что

$d$-мерное дискретное уравнение обладает свойством совместноcmu, если оно может быть согласованно распространено на все $d$-мерные подрешетки в $(d+1)$-мерной решетке

(более точное определение дается ниже). Из сказанного выше следует, что связь данного понятия с интегрируемостью не является новой идеей. В случае $d=1$ она использовалась как возможное определение интегрируемости отображений [24]. При $d=2$ решающий шаг был сделан в [10] и, независимо, в [19]: было

*Работа первого автора поддержана DFG Research Unit 565 "Polyhedral Surfaces" и грантом РФФИ 04-01-00403. Работа второго автора поддержана DFG Research Unit 565 "Polyhedral Surfaces". Работа третьего автора поддержана ESF Scientific Programme "Methods of Integrable Systems, Geometry, Applied Mathematics" (MISGAM). 
показано, что интегрируемость в обычном смысле солитонной теории (как существование представления нулевой кривизны) выводится для двумерных систем из трехмерной совместности. Таким образом, последнее свойство может быть принято в качестве определения интегрируемости. Данный критерий допускает алгоритмическую проверку, без какой-либо дополнительной информации, кроме той, что заложена в самом уравнении. Более того, если результат проверки положителен, то мы получаем также дискретное представление нулевой кривизны.

Элементарным строительным блоком систем на квад-графах является квадуравнение, т. е. уравнение

$$
Q\left(x_{1}, x_{2}, x_{3}, x_{4}\right)=0
$$

на четырехугольной ячейке, где полевые переменные $x_{i} \in \mathbb{C P}^{1}$ приписаны вершинам четырехугольника, как показано на рис. 1 . На решетке $\mathbb{Z}^{2}$ уравнения такого типа можно интерпретировать как дискретные аналоги нелинейных гиперболических уравнений. Начально-краевые задачи типа Гурса для таких систем изучались в [6].

Предположение. В данной работе мы предполагаем, что $Q$ является мультиаффинным многочленом, т. е. имеет степень 1 по каждому аргументу. Отсюда следует, что уравнение (1) можно разрешить относительно любой переменной, причем ответ является рациональной функцией от остальных трех переменных.

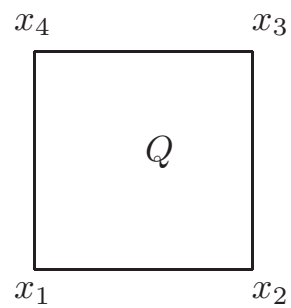

Рис. 1. Квад-уравнение $Q\left(x_{1}, x_{2}, x_{3}, x_{4}\right)=0$; переменные $x_{i}$ приписаны вершинам

Общая идея интегрируемости как совместности изображается для уравнений данного типа рисунком 2. Мы приписываем шесть квад-уравнений граням координатного куба. Значок $j$ обозначает сдвиг в направлении $j$-й координаты. Стартуя с произвольных значений $x, x_{1}, x_{2}, x_{3}$, мы находим значения $x_{12}, x_{13}$, $x_{23}$ при помощи трех уравнений на левой, передней и нижней гранях, а уравнения на правой, задней и верхней грани определяют, вообще говоря, три разных значения для $x_{123}$. Система называется $3 \mathrm{D}$-совместной, если данные три значения тождественно равны для произвольных начальных данных $x, x_{1}, x_{2}, x_{3}$.

В [3] мы классифицировали 3D-совместные системы частного вида. Уравнения на всех гранях предполагались одинаковыми с точностью до параметров, ассоциированных с тремя направлениями ребер. Кроме того, мы накладывали условия симметрии куба, а также некое дополнительное условие, так называемое свойство тетраэдральности (см. ниже). 3D-совместная система без этого свойства была найдена в [13], позднее было показано, что она линеаризуема [22]. 


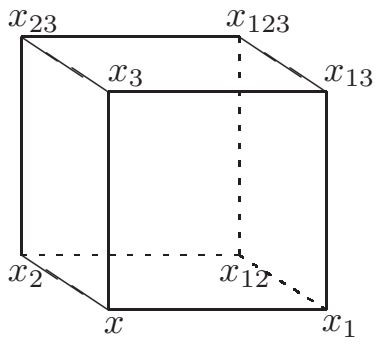

Рис. 2. 3D-совместная система квад-уравнений.

Уравнения приписаны граням куба

B [26] было показано, что 3D-совместные уравнения из списка [3] удовлетворяют тесту на интегрируемость, основанному на понятии алгебраической энтропии.

Данный подход обобщался в различных направлениях. Системы с полями на ребрах ведут к отображениям Янга-Бакстера [25], [23], [20]. Квадрирациональные отображения Янга-Бакстера были классифицированы в [4]. 4D-совместность дискретных 3D-систем связана с функциональным уравнением тетраэдра, изучавшимся в [17], [16], [15], [8].

В настоящей статье мы возвращаемся к задаче классификации 3D-совместных мультиаффинных квад-уравнений в более общей постановке. Допускается a priori, что граням совместного куба могут отвечать разные квад-уравнения. Не накладываются ни условие симметрии, ни условие тетраэдральности. Это ведет к общей классификации интегрируемых квад-уравнений.

Схематически наш подход заключается в следующем.

а) Последовательно исключая переменные при помощи операций типа дискриминанта, можно устроить спуск от мультиаффинного многочлена от четыpeх переменных, ассоциированного с четырехугольником, к биквадратичным многочленам от двух переменных, ассоциированным с его сторонами, и, наконец, к многочленам четвертой степени от одной переменной, ассоциированным с вершинами $(\S 2)$.

b) Анализируя сингулярные решения, мы доказываем, что биквадратичные многочлены, пришедшие на ребро куба с двух смежных граней, совпадают с точностью до постоянного множителя (см. §3). В этом месте требуется некоторое дополнительное условие невырожденности: мы предполагаем, что рассматриваемые биквадратичные многочлены не имеют множителей типа $x-$ const. (Примеры уравнений без этого свойства приведены в §7).

с) Это позволяет сопоставить каждой вершине куба многочлен четвертой степени от соответствующей переменной; допустимые наборы многочленов классифицируются по модулю преобразований Мёбиуса, причем каждая переменная преобразуется независимо от других (§4).

d) В завершение мы обращаем процедуру, восстанавливая сначала биквадратичные многочлены на ребрах куба, а затем и сами мультиаффинные уравнения $(\S 6)$. 


\section{§2. Мультиаффинные и биквадратичные уравнения}

Наш подход основан на спуске с граней на ребра и далее на вершины куба. В этом параграфе мы рассмотрим одну отдельную грань и опишем этот спуск безотносительно к 3D-совместности. Пусть $\mathscr{P}_{n}^{m}$ обозначает множество многочленов от $n$ переменных степени $m$ по каждой из них. Рассмотрим следующее действие преобразований Мёбиуса на многочлены $f \in \mathscr{P}_{n}^{m}$ :

$$
M[f]\left(x_{1}, \ldots, x_{n}\right)=\left(c_{1} x_{1}+d_{1}\right)^{m} \cdots\left(c_{n} x_{n}+d_{n}\right)^{m} f\left(\frac{a_{1} x_{1}+b_{1}}{c_{1} x_{1}+d_{1}}, \ldots, \frac{a_{n} x_{n}+b_{n}}{c_{n} x_{n}+d_{n}}\right),
$$

где $a_{i} d_{i}-b_{i} c_{i}=\Delta_{i} \neq 0$. Операции

$$
\mathscr{P}_{4}^{1} \stackrel{\delta_{x_{i}, x_{j}}}{\longrightarrow} \mathscr{P}_{2}^{2} \stackrel{\delta_{x_{k}}}{\longrightarrow} \mathscr{P}_{1}^{4}, \quad \delta_{x, y}(Q)=Q_{x} Q_{y}-Q Q_{x y}, \quad \delta_{x}(h)=h_{x}^{2}-2 h h_{x x},
$$

ковариантны по отношению к преобразованиям Мёбиуса (индексы $x, y$ обозначают частные производные). Точнее: если $Q \in \mathscr{P}_{4}^{1}, h \in \mathscr{P}_{2}^{2}$, то

$$
\delta_{x_{i}, x_{j}}(M[Q])=\Delta_{i} \Delta_{j} M\left[\delta_{x_{i}, x_{j}}(Q)\right], \quad \delta_{x_{i}}(M[h])=\Delta_{i}^{2} M\left[\delta_{x_{i}}(h)\right] .
$$

В дальнейшем мы широко используем относительные инварианты многочленов относительно преобразований Мёбиуса. Для многочленов четвертой степени $r \in \mathscr{P}_{1}^{4}$ эти относительные инварианты хорошо известны и определяются как коэффициенты вейерштрассовской нормальной формы $r=4 x^{3}-g_{2} x-g_{3}$. Для данного многочлена $r(x)=r_{4} x^{4}+r_{3} x^{3}+r_{2} x^{2}+r_{1} x+r_{0}$ они задаются формулами (см., например, [28])

$$
\begin{aligned}
g_{2}(r, x) & =\frac{1}{48}\left(2 r r^{I V}-2 r^{\prime} r^{\prime \prime \prime}+\left(r^{\prime \prime}\right)^{2}\right)=\frac{1}{12}\left(12 r_{0} r_{4}-3 r_{1} r_{3}+r_{2}^{2}\right), \\
g_{3}(r, x) & =\frac{1}{3456}\left(12 r r^{\prime \prime} r^{I V}-9\left(r^{\prime}\right)^{2} r^{I V}-6 r\left(r^{\prime \prime \prime}\right)^{2}+6 r^{\prime} r^{\prime \prime} r^{\prime \prime \prime}-2\left(r^{\prime \prime}\right)^{3}\right) \\
& =\frac{1}{432}\left(72 r_{0} r_{2} r_{4}-27 r_{1}^{2} r_{4}+9 r_{1} r_{2} r_{3}-27 r_{0} r_{3}^{2}-2 r_{2}^{3}\right) .
\end{aligned}
$$

При мёбиусовой замене $x=x_{1}$ эти величины лишь умножаются на постоянные множители:

$$
g_{k}(M[r], x)=\Delta_{1}^{2 k} g_{k}(r, x), \quad k=2,3 .
$$

Для биквадратичных многочленов $h \in \mathscr{P}_{2}^{2}$,

$$
h(x, y)=h_{22} x^{2} y^{2}+h_{21} x^{2} y+h_{20} x^{2}+h_{12} x y^{2}+h_{11} x y+h_{10} x+h_{02} y^{2}+h_{01} y+h_{00},
$$

относительными инвариантами являются величины

$$
\begin{aligned}
i_{2}(h, x, y) & =2 h h_{x x y y}-2 h_{x} h_{x y y}-2 h_{y} h_{x x y}+2 h_{x x} h_{y y}+h_{x y}^{2} \\
& =8 h_{00} h_{22}-4 h_{01} h_{21}-4 h_{10} h_{12}+8 h_{02} h_{20}+h_{11}^{2}, \\
i_{3}(h, x, y) & =\frac{1}{4} \operatorname{det}\left(\begin{array}{ccc}
h & h_{x} & h_{x x} \\
h_{y} & h_{x y} & h_{x x y} \\
h_{y y} & h_{x y y} & h_{x x y y}
\end{array}\right)=\operatorname{det}\left(\begin{array}{lll}
h_{22} & h_{21} & h_{20} \\
h_{12} & h_{11} & h_{10} \\
h_{02} & h_{01} & h_{00}
\end{array}\right) .
\end{aligned}
$$

Заметим, что $i_{3}$ можно определить также формулой

$$
-4 i_{3}(h, x, y)=\delta_{x, y}\left(\delta_{x, y}(h)\right) / h .
$$


Закон преобразования при мёбиусовых заменах $x=x_{1}$ и $y=x_{2}$ имеет вид

$$
i_{k}(M[h], x, y)=\Delta_{1}^{k} \Delta_{2}^{k} i_{k}(h, x, y), \quad k=2,3 .
$$

Следующие свойства операций $\delta_{x, y}, \delta_{x}$ проверяются прямыми вычислениями.

Лемма 1. Для любого мультиафбинного многочлена $Q\left(x_{1}, x_{2}, x_{3}, x_{4}\right) \in \mathscr{P}_{4}^{1}$ выполняются тождества

$$
\begin{aligned}
\delta_{x_{3}}\left(\delta_{x_{1}, x_{2}}(Q)\right) & =\delta_{x_{2}}\left(\delta_{x_{1}, x_{3}}(Q)\right), \\
i_{k}\left(\delta_{x_{1}, x_{2}}(Q), x_{3}, x_{4}\right) & =i_{k}\left(\delta_{x_{3}, x_{4}}(Q), x_{1}, x_{2}\right), \quad k=2,3 .
\end{aligned}
$$

Для любого биквадратичного многочлена $h\left(x_{1}, x_{2}\right) \in \mathscr{P}_{2}^{2}$ верно тожсдество

$$
g_{k}\left(\delta_{x_{1}}(h), x_{2}\right)=g_{k}\left(\delta_{x_{2}}(h), x_{1}\right), \quad k=2,3 .
$$

Положим $h^{i j}=h^{j i}=\delta_{x_{k}, x_{l}}(Q)$, где $\{i, j, k, l\}=\{1,2,3,4\}$. Тогда из леммы 1 следует коммутативность диаграммы

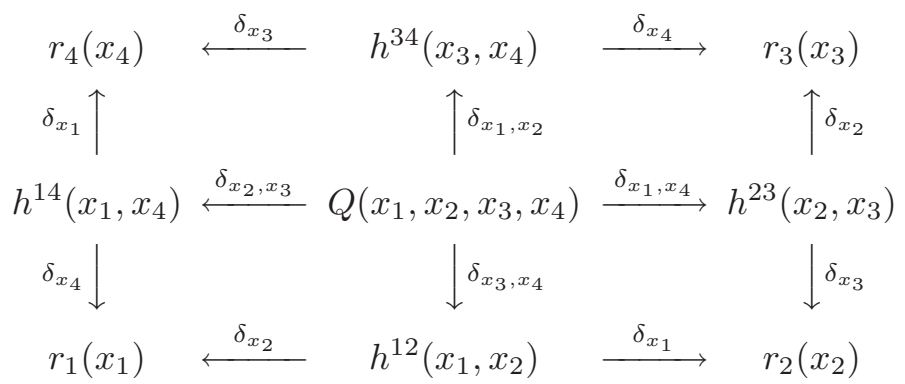

Более того, биквадратичные многочлены на противоположных сторонах имеют одинаковые инварианты $i_{2}, i_{3}$, а инварианты $g_{2}, g_{3}$ совпадают для всех многочленов четвертой степени $r_{i}$. Данная диаграмма может быть дополнена многочленами $h^{13}, h^{24}$, отвечающими диагоналям (так что возникает граф тетраэдра), но они нам не понадобятся. Для введенных многочленов выполняется еще ряд интересных соотношений.

Лемма 2. Для любого мультиафбинного многочлена $Q\left(x_{1}, x_{2}, x_{3}, x_{4}\right) \in \mathscr{P}_{4}^{1}$ выполнены следующие тождества (используются обозначения $h^{i j}\left(x_{i}, x_{j}\right)=$ $\left.\delta_{x_{k}, x_{l}}(Q) \in \mathscr{P}_{2}^{2}\right):$

$$
4 i_{3}\left(h^{12}, x_{1}, x_{2}\right) h^{14}=\operatorname{det}\left(\begin{array}{lll}
h^{12} & h_{x_{1}}^{12} & \ell \\
h_{x_{2}}^{12} & h_{x_{1} x_{2}}^{12} & \ell_{x_{2}} \\
h_{x_{2} x_{2}}^{12} & h_{x_{1} x_{2} x_{2}}^{12} & \ell_{x_{2} x_{2}}
\end{array}\right),
$$

где $\ell=h_{x_{3} x_{3}}^{23} h^{34}-h_{x_{3}}^{23} h_{x_{3}}^{34}+h^{23} h_{x_{3} x_{3}}^{34}$,

$$
\begin{gathered}
h^{12} h^{34}-h^{14} h^{23}=P Q, \quad P=\operatorname{det}\left(\begin{array}{lll}
Q & Q_{x_{1}} & Q_{x_{3}} \\
Q_{x_{2}} & Q_{x_{1} x_{2}} & Q_{x_{2} x_{3}} \\
Q_{x_{4}} & Q_{x_{1} x_{4}} & Q_{x_{3} x_{4}}
\end{array}\right) \in \mathscr{P}_{4}^{1}, \\
\frac{2 Q_{x_{1}}}{Q}=\frac{h_{x_{1}}^{12} h^{34}-h_{x_{1}}^{14} h^{23}+h^{23} h_{x_{3}}^{34}-h_{x_{3}}^{23} h^{34}}{h^{12} h^{34}-h^{14} h^{23}} .
\end{gathered}
$$


Тождество (8) показывает, что $h^{14}$ можно выразить через три остальных биквадратичных многочлена (при условии, что $i_{3}\left(h^{12}\right) \neq 0$ ). Тождество (9) определяет $Q$ как один из сомножителей в простом выражении, составленном из $h^{i j}$. Наконец, дифференцируя (10) по $x_{2}$ или $x_{4}$, мы получаем соотношение вида $Q^{2}=F\left[h^{12}, h^{23}, h^{34}, h^{14}\right]$, где $F$ есть рациональное выражение от $h^{i j}$ и их производных. Следовательно, если известны биквадратичные многочлены на трех сторонах из четырех, то $Q$ находится явно. Конечно, из леммы 2 ясно, что не всякий набор из трех биквадратичных многочленов может быть получен как $h^{i j}$ для некоторого $Q \in \mathscr{P}_{4}^{1}$.

Биквадратичные многочлены $h^{i j}$ для данного $Q \in \mathscr{P}_{4}^{1}$ тесно связаны с сингулярными решениями мультиаффинного уравнения

$$
Q\left(x_{1}, x_{2}, x_{3}, x_{4}\right)=0 .
$$

Многочлен $Q \in \mathscr{P}_{4}^{1}$ предполагается неприводимым (в частности, $Q_{x_{i}} \not \equiv 0$ : иначе многочлен $Q$ следует считать приводимым, так как замена $x_{i} \mapsto 1 / x_{i}$ переводит его в $\left.x_{i} Q\right)$. Очевидно, что уравнение (11) разрешимо относительно любой переменной: пусть $Q=p\left(x_{j}, x_{k}, x_{l}\right) x_{i}+q\left(x_{j}, x_{k}, x_{l}\right)$; тогда $x_{i}=-q / p$ для значений $x_{j}, x_{k}, x_{l}$ общего положения. Однако значение $x_{i}$ не определено в точках $\left(x_{j}, x_{k}, x_{l}\right)$, лежащих на кривой

$$
S_{i}: \quad p\left(x_{j}, x_{k}, x_{l}\right)=q\left(x_{j}, x_{k}, x_{l}\right)=0, \quad Q \equiv p x_{i}+q,
$$

в $\left(\mathbb{C P}^{1}\right)^{3}$. Проекция этой кривой на координатную плоскость $(j, k)$ есть в точности биквадратичная кривая $h^{j k}=p q_{x_{l}}-p_{x_{l}} q=0$.

Определение 1. Решение $\left(x_{1}, x_{2}, x_{3}, x_{4}\right)$ уравнения (11) называется сингулярным относительно $x_{i}$, если оно удовлетворяет также уравнению $Q_{x_{i}}\left(x_{1}, x_{2}\right.$, $\left.x_{3}, x_{4}\right)=0$. Кривая $S_{i}$ называется сингулярной кривой для $x_{i}$.

Лемма 3. Если решение $\left(x_{1}, x_{2}, x_{3}, x_{4}\right)$ уравнения (11) сингулярно относительно $x_{i}$, то $h^{j k}=h^{j l}=h^{k l}=0$. Обратно, если для некоторого решения $h^{j k}=0$, то это решение сингулярно либо относительно $x_{i}$, либо относительно $x_{l}$.

Доказательство. Так как $h^{j k}=Q_{x_{i}} Q_{x_{l}}-Q Q_{x_{i}, x_{l}}$, то уравнения $h^{j k}=0$ и $Q_{x_{i}} Q_{x_{l}}=0$ эквивалентны на решениях уравнения $Q=0$.

Мы используем следующее определение невырожденности биквадратичных многочленов.

Определение 2. Биквадратичный многочлен $h(x, y) \in \mathscr{P}_{2}^{2}$ называется невырожденным, если его класс эквивалентности по модулю преобразований Мёбиуса не содержит многочленов, делящихся на множитель вида $x-c$ или $y-c$, где $c=$ const.

Согласно данному определению, невырожденный многочлен $h(x, y) \in \mathscr{P}_{2}^{2}$ либо неприводим, либо имеет вид $\left(\alpha_{1} x y+\beta_{1} x+\gamma_{1} y+\delta_{1}\right)\left(\alpha_{2} x y+\beta_{2} x+\gamma_{2} y+\delta_{2}\right)$, где $\alpha_{i} \delta_{i} \neq \beta_{i} \gamma_{i}$. В обоих случаях уравнение $h=0$ определяет $y$ как двузначную функцию от $x$ и наоборот. С другой стороны, например, многочлен $h(x, y)=$ $x-y^{2}$ (рассматриваемый как элемент из $\mathscr{P}_{2}^{2}$ ) является, согласно определению 2 , вырожденным, так как инверсия $x \mapsto 1 / x$ переводит его в $x\left(1-x y^{2}\right)$.

Следующее понятие играет фундаментальную роль в нашем исследовании. 
Определение 3. Мультиаффинная функция $Q \in \mathscr{P}_{4}^{1}$ относится к типу $Q$, если невырожденны все четыре отвечающих ей биквадратичных многочлена $h^{j k} \in \mathscr{P}_{2}^{2}$, и к типу $H$ в противном случае.

\section{§3. 3D-совместность и биквадратичные кривые}

Рассмотрим систему уравнений

$$
\begin{array}{lll}
A\left(x, x_{1}, x_{2}, x_{12}\right)=0, & \bar{A}\left(x_{3}, x_{13}, x_{23}, x_{123}\right)=0, \\
B\left(x, x_{1}, x_{3}, x_{13}\right)=0, & \bar{B}\left(x_{2}, x_{12}, x_{23}, x_{123}\right)=0, \\
C\left(x, x_{2}, x_{3}, x_{23}\right)=0, & \bar{C}\left(x_{1}, x_{12}, x_{13}, x_{123}\right)=0
\end{array}
$$

на кубе, см. рис. 3. Функции $A, \ldots, \bar{C}$ считаются мультиаффинными (т. е. принадлежат $\left.\mathscr{P}_{4}^{1}\right)$ и a priori никак не связаны друг с другом. Для соответствующих биквадратичных многочленов мы будем использовать обозначение $A^{i j}=$ $\delta_{x_{k}, x_{l}} A$.

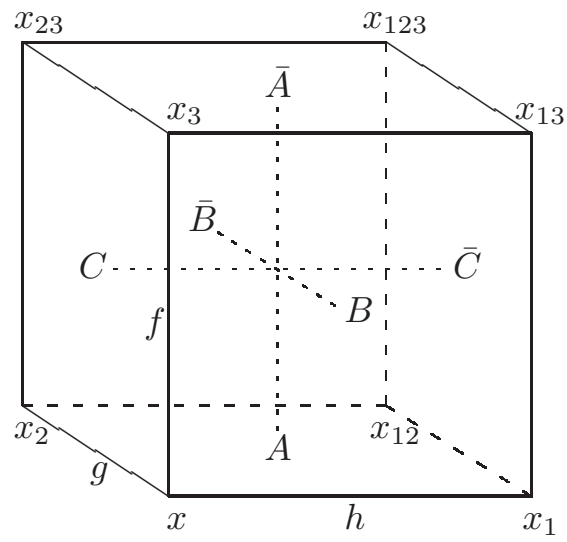

Рис. 3. 3D-совместная система квад-уравнений. Уравнения ассоциированы с гранями куба: $A$ и $\bar{A}$ с нижней и верхней, $B$ и $\bar{B}$ с передней и задней, $C$ и $\bar{C}$ с левой и правой.

Теорема 1. Пусть все шесть функиий $A, \ldots, \bar{C}$ относятся $\kappa$ mипу $Q, u$ пусть система (13) 3D-совместна. Тогда

1) для каждого ребра куба два биквадратичных многочлена, отвечающих этому ребру (пришедиих с двух граней, смежных по данному ребру), совпадают с точностъю до постоянного множителя;

2) произведение этих множителей вокруг любой вершины равно -1, например,

$$
A^{0,1} B^{0,3} C^{0,2}+A^{0,2} B^{0,1} C^{0,3}=0 ;
$$

3) система (13) обладает свойством тетраэдральности $\partial x_{123} / \partial x=0$.

Доказательство. Исключение $x_{12}, x_{13}$ и $x_{23}$ приводит к уравнениям $F\left(\stackrel{2}{x}, \stackrel{1}{x}_{1}, \stackrel{1}{x_{2}}, \stackrel{3}{x_{3}}, \stackrel{1}{x_{123}}\right)=\bar{A}_{x_{13}, x_{23}} B C-\bar{A}_{x_{23}} B_{x_{13}} C-\bar{A}_{x_{13}} B C_{x_{23}}+\bar{A} B_{x_{13}} C_{x_{23}}=0$, $G\left(\stackrel{2}{x}, \stackrel{1}{x}_{1}, \stackrel{3}{x_{2}}, \stackrel{1}{x_{3}}, \stackrel{1}{x}_{123}\right)=\bar{B}_{x_{12}, x_{23}} A C-\bar{B}_{x_{23}} A_{x_{12}} C-\bar{B}_{x_{12}} A C_{x_{23}}+\bar{B} A_{x_{12}} C_{x_{23}}=0$, $H\left(\stackrel{2}{x}, \stackrel{3}{x}_{1}, \stackrel{1}{x}_{2}, \stackrel{1}{x}_{3}, \stackrel{1}{x}_{123}\right)=\bar{C}_{x_{12}, x_{13}} A B-\bar{C}_{x_{13}} A_{x_{12}} B-\bar{C}_{x_{12}} A B_{x_{13}}+\bar{C} A_{x_{12}} B_{x_{13}}=0$. 
Здесь числа над аргументами функций $F, G, H$ указывают степень, с которой данная переменная входит в правую часть (степень понимается в проективном смысле, см. пример в конце предыдущего параграфа). В силу 3D-совместности выражения для $x_{123}$ как функции от $x, x_{1}, x_{2}, x_{3}$, найденные из этих уравнений, должны совпадать. Следовательно, имеют место разложения на множители

$$
F=f\left(x, \stackrel{2}{x}_{3}\right) K, \quad G=g\left(x, \stackrel{2}{x}_{2}\right) K, \quad H=h\left(x, \stackrel{2}{x}_{1}\right) K, \quad K=K\left(x, \stackrel{1}{x}_{1}, \stackrel{1}{x}_{2}, \stackrel{1}{x}_{3}, \stackrel{1}{x}_{123}\right),
$$

где $f, g, h$ - некие многочлены степени 2 по второму аргументу. Степень по $x$ следует уточнить.

Пусть начальные значения $x, x_{1}, x_{2}$ суть свободные переменные, а $x_{3}$ выберем так, чтобы выполнялось уравнение $f\left(x, x_{3}\right)=0$. Тогда $F \equiv 0$ и, следовательно, система $B=C=\bar{A}=0$ не дает определенного значения $x_{123}$. При этом уравнение $B=0$ разрешимо относительно $x_{13}$, так как иначе начальные данные были бы связаны соотношением $B^{0,1}\left(x, x_{1}\right)=0$. Аналогично, уравнение $C=0$ разрешимо относительно $x_{23}$. Следовательно, неопределенность возникает из-за сингулярности уравнения $\bar{A}=0$ относительно $x_{123}$. Следовательно, выполняется связь $\bar{A}^{3,13}\left(x_{3}, x_{13}\right)=0$. В силу предположения теоремы $x_{13}$ является (двузначной) функцией от $x_{3}$ и не зависит от $x_{1}$. Это значит, что уравнение $B=0$ сингулярно относительно $x_{1}$ и, следовательно, $B^{0,3}\left(x, x_{3}\right)=0$. Аналогично, $C^{0,3}\left(x, x_{3}\right)=0$.

Итак, мы доказали, что если $x_{3}=\varphi(x)$ есть нуль многочлена $f$, то он является также нулем многочленов $B^{0,3}$ и $C^{0,3}$. Если один из этих трех многочленов неприводим, то отсюда уже следует их совпадение с точностью до постоянного множителя. Если многочлены приводимы, мы не можем этого утверждать, так как имеется возможность $f=a^{2}, B^{0,3}=a b, C^{0,3}=a c$, где $a, b, c$ мультиаффинны по $x, x_{3}$. Однако в любом случае $\operatorname{deg}_{x} f=2$, и этого достаточно для завершения доказательства.

Действительно, отсюда следует, что $\operatorname{deg}_{x} K=0$, т. е. выполняется свойство тетраэдральности. В свою очередь, из этого вытекает соотношение (14), как показано в [3]. Напомним это вычисление: перепишем систему (13) в виде

$$
\begin{gathered}
x_{12}=a\left(x, x_{1}, x_{2}\right), \quad x_{13}=b\left(x, x_{1}, x_{3}\right), \quad x_{23}=c\left(x, x_{2}, x_{3}\right), \\
x_{123}=d\left(x_{1}, x_{2}, x_{3}\right)=\bar{a}\left(x_{3}, x_{13}, x_{23}\right)=\bar{b}\left(x_{2}, x_{12}, x_{23}\right)=\bar{c}\left(x_{1}, x_{12}, x_{13}\right)
\end{gathered}
$$

и получим дифференцированием

$$
\begin{array}{lll}
d_{x_{1}}=\bar{a}_{x_{13}} b_{x_{1}}, & d_{x_{2}}=\bar{a}_{x_{23}} c_{x_{2}}, & 0=\bar{a}_{x_{13}} b_{x}+\bar{a}_{x_{23}} c_{x}, \\
d_{x_{1}}=\bar{b}_{x_{12}} a_{x_{1}}, & d_{x_{3}}=\bar{b}_{x_{23}} c_{x_{3}}, & 0=\bar{b}_{x_{12}} a_{x}+\bar{b}_{x_{23}} c_{x}, \\
d_{x_{2}}=\bar{c}_{x_{12}} a_{x_{2}}, & d_{x_{3}}=\bar{c}_{x_{13}} b_{x_{3}}, & 0=\bar{c}_{x_{12}} a_{x}+\bar{c}_{x_{13}} b_{x} .
\end{array}
$$

Эти уравнения сразу дают соотношение

$$
a_{x_{2}} b_{x_{1}} c_{x_{3}}+a_{x_{1}} b_{x_{3}} c_{x_{2}}=0
$$

эквивалентное (14) в силу тождества $a_{x_{2}} / a_{x_{1}}=A^{0,1} / A^{0,2}$. Переменные в уравнении (14) разделяются: $B^{0,3} / C^{0,3}=-A^{0,2} / C^{0,2} \cdot B^{0,1} / A^{0,1}$, так что $B^{0,3} / C^{0,3}$ может зависеть лишь от $x$. В силу предположения теоремы это отношение постоянно. 
Существуют 3D-совместные системы с уравнениями, не принадлежащими типу $Q$. Для таких систем утверждения теоремы 1 могут выполняться или не выполняться, как показывают следующие примеры.

Пример 1. Простейшим 3D-совместным уравнением является линейное уравнение

$$
x+x_{i}+x_{j}+x_{i j}=0 .
$$

В этом случае все биквадратичные многочлены равны 1, так что утверждение 1) выполнено, а утверждение 2) - нет. Так как 2) есть следствие свойства тетраэдральности 3), то последнее также не выполняется. Действительно,

$$
x_{123}=2 x+x_{1}+x_{2}+x_{3} .
$$

Множитель $f$ в этом примере также равен 1, но это совпадение нарушается при преобразованиях Мёбиуса. Действительно, в этом случае $\operatorname{deg}_{x} K=1$, и после инверсии всех переменных $x_{I} \rightarrow 1 / x_{I}$ мы приходим к $f=x x_{3}^{2}$, тогда как $B^{0,3}$ переходит в $x^{2} x_{3}^{2}$.

Пример 2. Уравнение Хиетаринты [13]

$$
\left(x-e^{(j)}\right)\left(x_{i j}-o^{(j)}\right)\left(x_{i}-o^{(i)}\right)\left(x_{j}-e^{(i)}\right)-\left(x-e^{(i)}\right)\left(x_{i j}-o^{(i)}\right)\left(x_{i}-e^{(j)}\right)\left(x_{j}-o^{(j)}\right)=0
$$

3D-совместно, но утверждение 1) не выполняется:

$$
\begin{aligned}
& B^{0,3}=\left(e^{(3)}-o^{(1)}\right)\left(o^{(1)}-o^{(3)}\right)\left(x-e^{(3)}\right)\left(x-e^{(1)}\right)\left(x_{3}-e^{(1)}\right)\left(x_{3}-o^{(3)}\right), \\
& C^{0,3}=\left(e^{(3)}-o^{(2)}\right)\left(o^{(2)}-o^{(3)}\right)\left(x-e^{(3)}\right)\left(x-e^{(2)}\right)\left(x_{3}-e^{(2)}\right)\left(x_{3}-o^{(3)}\right) .
\end{aligned}
$$

Множитель $f$ пропорционален $\left(x-e^{(3)}\right)\left(x_{3}-e^{(1)}\right)\left(x_{3}-e^{(2)}\right)$. Соответственно $\operatorname{deg}_{x} K=1$ и свойство тетраэдральности не выполняется.

Пример 3. Вероятно, самый известный пример 3D-совместной системы это дискретное потенциальное уравнение $\mathrm{KdV}$

$$
\left(x-x_{i j}\right)\left(x_{i}-x_{j}\right)+\alpha^{(i)}-\alpha^{(j)}=0 .
$$

В этом случае все утверждения теоремы выполняются, несмотря на вырожденность биквадратичных кривых:

$$
B^{0,3}=\alpha^{(1)}-\alpha^{(3)}, \quad C^{0,3}=\alpha^{(2)}-\alpha^{(3)}, \quad f=1 .
$$

(Напомним, что степень понимается в проективном смысле. При инверсии эти многочлены превращаются в $x^{2} x_{3}^{2}$.)

Пример 4. Уравнение $\left(Q_{1}\right)$

$$
\begin{aligned}
& Q\left(x, x_{1}, x_{2}, x_{12} ; \alpha^{(1)}, \alpha^{(2)} ; \delta\right) \\
& =\alpha^{(1)}\left(x-x_{2}\right)\left(x_{1}-x_{12}\right)-\alpha^{(2)}\left(x-x_{1}\right)\left(x_{2}-x_{12}\right)+\delta \alpha^{(1)} \alpha^{(2)}\left(\alpha^{(1)}-\alpha^{(2)}\right)=0
\end{aligned}
$$

составляет совместную систему не только с собственными копиями (см. [3] и теорему 4 ниже), но и с линейными уравнениями. А именно, система, образованная уравнениями

$$
Q\left(x, x_{1}, x_{12}, x_{2} ; \alpha^{(1)}, \alpha^{(2)} ; \delta\right)=0, \quad x_{13}-x_{3}=x_{1}-x, \quad x_{23}-x_{3}=x_{2}-x
$$


и их копиями на противоположных гранях, оказывается 3D-совместной. В этом случае ребру $\left(x, x_{3}\right)$ отвечают многочлены

$$
B^{0,3}=C^{0,3}=-1, \quad f=1 .
$$

Однако, в отличие от предыдущего примера, свойство тетраэдральности не выполняется и $\operatorname{deg}_{x} K=2$. Это значит, что многочлен $f$ не биквадратичен и его образ при инверсии есть $x_{3}^{2}$. Более того, биквадратичные многочлены, отвечающие ребру $\left(x, x_{1}\right)$, не совпадают:

$$
A^{0,1}=\alpha^{(2)}\left(\alpha^{(1)}-\alpha^{(2)}\right)\left(\left(x_{1}-x\right)^{2}-\delta\left(\alpha^{(1)}\right)^{2}\right), \quad B^{0,1}=-1, \quad h=1 .
$$

В этом примере мы видим, что возможна ситуация, когда предположения теоремы выполняются для одной части биквадратичных многочленов и не выполняются для другой.

\section{§4. Классификация биквадратичных многочленов}

Диаграмма (7) подсказывает алгоритм классификации мультиаффинных уравнений $Q=0$ по модулю преобразований Мёбиуса. Первый шаг заключается в том, чтобы использовать преобразования Мёбиуса для приведения многочленов $r_{i}\left(x_{i}\right)$, ассоциированных с вершинами четырехугольника, к канонической форме. Согласно формулам (2),

$$
\delta_{x_{l}}\left(\delta_{x_{j}, x_{k}}(M[Q])\right)=\Delta_{j}^{2} \Delta_{k}^{2} \Delta_{l}^{2} M\left[\delta_{x_{l}}\left(\delta_{x_{j}, x_{k}}(Q)\right)\right]=\frac{C}{\Delta_{i}^{2}} M\left[r_{i}\right],
$$

где $C=\Delta_{1}^{2} \Delta_{2}^{2} \Delta_{3}^{2} \Delta_{4}^{2}$. Так как многочлен $Q$ определен с точностью до произвольного множителя, мы можем считать, что мёбиусовы замены переменных в уравнении $Q=0$ индуцируют преобразования

$$
r_{i} \mapsto \frac{1}{\Delta_{i}^{2}} M\left[r_{i}\right]
$$

многочленов $r_{i}$. Это позволяет привести каждый из $r_{i}$ к одной из следующих форм:

$$
r=\left(x^{2}-1\right)\left(k^{2} x^{2}-1\right), \quad r=x^{2}-1, \quad r=x^{2}, \quad r=x, \quad r=1, \quad r=0,
$$

согласно следующим шести возможностям: $r$ имеет четыре простых корня, два простых корня и один двойной, два двойных корня, простой и тройной корни, корень кратности четыре и, наконец, $r$ равен нулю тождественно. В первой канонической форме мы всегда будем предполагать, что $k \neq 0, \pm 1$, так что вторая и третья формы не считаются частным случаем первой.

Не всякая пара многочленов может отвечать смежным вершинам, так как относительные инварианты многочленов в такой паре должны совпадать согласно (6). Мы определим все допустимые пары, а также решим задачу восстановления биквадратичного многочлена (3) по паре его дискриминантов

$$
\delta_{y}(h)=h_{y}^{2}-2 h h_{y y}=r_{1}(x), \quad \delta_{x}(h)=h_{x}^{2}-2 h h_{x x}=r_{2}(y),
$$

что равносильно решению системы из 10 (билинейных) уравнений для 9 неизвестных коэффициентов многочлена $h$. 
Теорема 2. Биквадратичные многочлены с парой дискриминантов $\left(r_{1}(x)\right.$, $\left.r_{2}(y)\right)$ в канонической форме существуют для следующих пар, с точностью до перестановки $x, y$ :

\begin{tabular}{|c|c|c|c|c|c|c|}
\hline & $\left(y^{2}-1\right)\left(k^{2} y^{2}-1\right)$ & $y^{2}-1$ & $y^{2}$ & $y$ & 1 & 0 \\
\hline$\left(x^{2}-1\right)\left(k^{2} x^{2}-1\right)$ & + & & & & & \\
\hline$x^{2}-1$ & & + & + & & & \\
\hline$x^{2}$ & & & + & & & \\
\hline$x$ & & & & + & + & \\
\hline 1 & & & & & + & + \\
\hline 0 & & & & & & + \\
\hline
\end{tabular}

Эти многочлены $h$ и их относительные инварианты $i_{2}, i_{3}$ перечислены в следуюшем списке:

$$
\begin{aligned}
& (r(x), r(y)), r(x)=\left(x^{2}-1\right)\left(k^{2} x^{2}-1\right): \\
& h=\frac{1}{2 \alpha}\left(k^{2} \alpha^{2} x^{2} y^{2}+2 A x y-x^{2}-y^{2}+\alpha^{2}\right), \quad A^{2}=r(\alpha), \\
& i_{2}=3\left(k^{2} \alpha^{2}+\alpha^{-2}\right)-k^{2}-1, \quad 4 i_{3}=A\left(k^{2} \alpha-\alpha^{-3}\right) \\
& \left(x^{2}-\delta, y^{2}-\delta\right): \quad h=\frac{\alpha}{1-\alpha^{2}}\left(x^{2}+y^{2}\right)-\frac{1+\alpha^{2}}{1-\alpha^{2}} x y+\frac{\delta\left(1-\alpha^{2}\right)}{4 \alpha}, \\
& i_{2}=\frac{1+10 \alpha^{2}+\alpha^{4}}{\left(1-\alpha^{2}\right)^{2}}, \quad i_{3}=\frac{\alpha^{2}\left(1+\alpha^{2}\right)}{\left(1-\alpha^{2}\right)^{3}}
\end{aligned}
$$

$(x, y): \quad h=\frac{1}{4 \alpha}(x-y)^{2}-\frac{\alpha}{2}(x+y)+\frac{\alpha^{3}}{4}, \quad i_{2}=\frac{3}{4 \alpha^{2}}, i_{3}=\frac{1}{32 \alpha^{3}} ;$

$\left(x^{2}, y^{2}\right): \quad h=\lambda x^{2}+\mu x y+\nu y^{2}, \quad \mu^{2}-4 \lambda \nu=1, i_{2}=1+12 \lambda \nu, i_{3}=-\lambda \mu \nu ;$

$$
h=\lambda x^{2} y^{2}+\mu x y+\nu, \quad \mu^{2}-4 \lambda \nu=1, i_{2}=1+12 \lambda \nu, i_{3}=\lambda \mu \nu
$$

$(1,1): \quad h=\lambda(x \pm y)^{2}+\mu(x \pm y)+\nu, \quad \mu^{2}-4 \lambda \nu=1, i_{2}=12 \lambda^{2}, i_{3}=\mp 2 \lambda^{3}$;

$(0,0): \quad h=(\varkappa x y+\lambda x+\mu y+\nu)^{2}, \quad i_{2}=12(\varkappa \nu-\lambda \mu)^{2}, i_{3}=2(\varkappa \nu-\lambda \mu)^{3}$;

$\left(x^{2}-1, y^{2}\right): \quad h=\alpha y^{2} \pm x y+\frac{1}{4 \alpha}, \quad i_{2}=1, i_{3}=0 ;$

$(x, 1): \quad h= \pm \frac{1}{4}(y-\alpha)^{2} \mp x, \quad i_{2}=0, i_{3}=0 ;$

$(1,0): \quad h=\lambda y^{2}+\mu y+\nu, \quad \mu^{2}-4 \lambda \nu=1, i_{2}=0, i_{3}=0$.

Доказательство. Список получен прямым решением системы (18) для различных канонических пар $\left(r_{1}, r_{2}\right)$. Перебор сокращается, если заметить, что $g_{2}^{3} \neq 27 g_{3}^{2}$ только в одном случае и что относительные инварианты для многочлена $r_{1}=a x^{2}+b x+c$ суть $12 g_{2}=a^{2}, 216 g_{3}=-a^{3}$, так что второй многочлен должен иметь вид $r_{2}=a y^{2}+\tilde{b} y+\tilde{c}$. Решение для пары $(x, 0)$ оказывается пустым. 


\section{§5. Классификация мультиаффинных уравнений типа $Q$}

Важно отметить, что после приведения многочленов $r_{i}\left(x_{i}\right)$ к каноническому виду еще остается некоторая свобода. А именно, можно применять преобразования Мёбиуса, не меняющие вид $r$, для дальнейшего упрощения биквадратичной кривой $h$ и мультиаффинного уравнения $Q$. В частности, список из теоремы 2 является более детальным, чем просто список биквадратичных многочленов по модулю преобразований Мёбиуса.

Действительно, многочлен (22) превращается в (23) при инверсии $x$; замена $x \mapsto-x$ позволяет зафиксировать знаки в многочленах (24), (26); в случае $(27)$ знак фиксируется заменой $x \mapsto-x, y \mapsto i y$; многочлены (25), (28) допускают дальнейшее упрощение.

Однако преобразование переменной, отвечающей одной из четырех вершин четырехугольника, влияет на биквадратичные многочлены, отвечающие двум смежным сторонам, и, следовательно, a priori нельзя гарантировать, что все четыре биквадратичные кривые можно привести к некоторой определенной форме одновременно. Например, если каждой вершине отвечает многочлен $r_{i}=x_{i}^{2}$, то ребрам могут отвечать многочлены вида (22) или вида (23). Заранее не известно, можно ли привести все эти многочлены к одному и тому же виду (хотя бы и с разными коэффициентами). На самом деле это возможно, как показывает доказательство следующей теоремы.

Следующий шаг заключается в восстановлении мультиаффинных многочленов по биквадратичным. Так как нашей целью является классификация систем только типа $Q$, нам не нужно решать эту задачу во всей полноте. Мы отбрасываем случаи $(26),(27)$ и (28), так как соответствующие биквадратичные кривые вырожденны. По этой же причине накладываются дополнительные ограничения на значения параметров: $\lambda \nu \neq 0$ в случаях (22), (23), $\lambda \neq 0$ в случае (24) и $\varkappa \nu-\lambda \mu \neq 0$ в случае $(25)$.

Теорема 3. Любое мультиаффинное уравнение типа $Q$ эквивалентно, с точностъю до преобразований Мёбиуса, одному из уравнений из следующего списка:

$$
\begin{aligned}
& \operatorname{sn}(\alpha) \operatorname{sn}(\beta) \operatorname{sn}(\alpha+\beta)\left(k^{2} x_{1} x_{2} x_{3} x_{4}+1\right)-\operatorname{sn}(\alpha)\left(x_{1} x_{2}+x_{3} x_{4}\right) \\
& -\operatorname{sn}(\beta)\left(x_{1} x_{4}+x_{2} x_{3}\right)+\operatorname{sn}(\alpha+\beta)\left(x_{1} x_{3}+x_{2} x_{4}\right)=0 \\
& \left(\alpha-\alpha^{-1}\right)\left(x_{1} x_{2}+x_{3} x_{4}\right)+\left(\beta-\beta^{-1}\right)\left(x_{1} x_{4}+x_{2} x_{3}\right)-\left(\alpha \beta-\alpha^{-1} \beta^{-1}\right)\left(x_{1} x_{3}+x_{2} x_{4}\right) \\
& \quad+\frac{\delta}{4}\left(\alpha-\alpha^{-1}\right)\left(\beta-\beta^{-1}\right)\left(\alpha \beta-\alpha^{-1} \beta^{-1}\right)=0 \\
& \alpha\left(x_{1}-x_{4}\right)\left(x_{2}-x_{3}\right)+\beta\left(x_{1}-x_{2}\right)\left(x_{4}-x_{3}\right) \\
& \quad-\alpha \beta(\alpha+\beta)\left(x_{1}+x_{2}+x_{3}+x_{4}\right)+\alpha \beta(\alpha+\beta)\left(\alpha^{2}+\alpha \beta+\beta^{2}\right)=0, \\
& \alpha\left(x_{1}-x_{4}\right)\left(x_{2}-x_{3}\right)+\beta\left(x_{1}-x_{2}\right)\left(x_{4}-x_{3}\right)-\delta \alpha \beta(\alpha+\beta)=0 .
\end{aligned}
$$

Доказательство. Пусть многочлены $h^{12}, h^{23}, h^{34}$ и $h^{14}$ имеют вид (19) с параметрами $(\alpha, A),(\beta, B),(\tilde{\alpha}, \tilde{A})$ и $(\tilde{\beta}, \tilde{B})$ соответственно, лежащими на эллиптической кривой $A^{2}=r(\alpha)$. Относительные инварианты $i_{2}, i_{3}$ многочленов $h^{12}$ и $h^{34}$ должны совпадать в силу (5), и легко убедиться, что это условие 
оставляет для $(\tilde{\alpha}, \tilde{A})$ только следующие возможные значения:

$$
(\alpha, A), \quad(-\alpha,-A), \quad \frac{1}{k \alpha^{2}}(\alpha,-A), \quad \frac{1}{k \alpha^{2}}(-\alpha, A),
$$

и аналогичные значения возможны для $(\tilde{\beta}, \tilde{B})$. На первый взгляд, нам предстоит перебор 16 четверок $h^{i j}$, но на самом деле ситуация значительно лучше. Действительно, согласно (2), при преобразовании Мёбиуса в уравнении $Q=0$ выполняются соотношения

$$
\delta_{x_{k}, x_{l}}(M[Q])=\Delta_{k} \Delta_{l} M\left[\delta_{x_{k}, x_{l}}(Q)\right]=\frac{C}{\Delta_{i} \Delta_{j}} M\left[h^{i j}\right],
$$

где $C=\Delta_{1} \Delta_{2} \Delta_{3} \Delta_{4}$. Так как $Q$ определен с точностью до умножения на константу, то можно считать, что мёбиусовы замены в уравнении $Q=0$ индуцируют преобразования

$$
h^{i j} \mapsto \frac{1}{\Delta_{i} \Delta_{j}} M\left[h^{i j}\right]
$$

биквадратичных многочленов $h^{i j}$. В частности, если

$$
h^{34}=h\left(x_{3}, x_{4},-\alpha,-A\right) \quad \text { или } \quad h^{34}=h\left(x_{3}, x_{4}, \frac{1}{k \alpha},-\frac{A}{k \alpha^{2}}\right),
$$

то преобразование $x_{3} \mapsto-x_{3}$, соответственно $x_{3} \mapsto 1 /\left(k x_{3}\right)$, приведет $h^{34}$ к виду

$$
-h\left(-x_{3}, x_{4} ;-\alpha,-A\right), \quad \text { соответственно }-k x_{3}^{2} h\left(\frac{1}{k x_{3}}, x_{4} ; \frac{1}{k \alpha},-\frac{A}{k \alpha^{2}}\right),
$$

что совпадает с $h\left(x_{3}, x_{4}, \alpha, A\right)$ в силу симметрий многочлена (19). Таким образом, сделав подходящую замену переменной $x_{3}$ (причем многочлен $r\left(x_{3}\right)$ не меняется), мы можем предполагать, не теряя общности, что $(\tilde{\alpha}, \tilde{A})=(\alpha, A)$. После этого многочлен $h^{14}$ однозначно определяется по формуле (8), и оказывается, что при этом автоматически выполнено равенство $(\tilde{\beta}, \tilde{B})=(\beta, B)$. Итак, замена одной переменной позволяет достичь совпадения параметров на противоположных сторонах квадрата. Прямое вычисление с использованием формулы (10) приводит к уравнению

$$
\alpha \beta \gamma\left(k^{2} x_{1} x_{2} x_{3} x_{4}+1\right)+\alpha\left(x_{1} x_{2}+x_{3} x_{4}\right)+\beta\left(x_{1} x_{4}+x_{2} x_{3}\right)+\gamma\left(x_{1} x_{3}+x_{2} x_{4}\right)=0,
$$

где $\gamma=(\alpha B+\beta A) /\left(k^{2} \alpha^{2} \beta^{2}-1\right)$, и, наконец, замена $\alpha \rightarrow \operatorname{sn}(\alpha), A \rightarrow \operatorname{sn}^{\prime}(\alpha)$ и аналогичная замена для $\beta$ приводят его к виду (29).

В других случаях подходящая мёбиусова замена переменных $x_{2}, x_{3}, x_{4}$ также позволяет привести многочлены к виду $h^{12}=h\left(x_{1}, x_{2}, \alpha\right), h^{23}=h\left(x_{2}, x_{3}, \beta\right)$, $h^{34}=h\left(x_{3}, x_{4}, \alpha\right)$. При этом прямое вычисление по формуле (8) доказывает, что, кроме того, $h^{14}=h\left(x_{1}, x_{4}, \beta\right)$. После этого ответ находится при помощи $(10)$.

Подробнее, многочлены (20) приводят к уравнению (30). В этом случае из уравнений (5) следует, что параметры $\alpha$ многочленов $h^{12}$ и $h^{34}$ отличаются не более чем знаком. Это компенсируется заменой $x_{3} \rightarrow-x_{3}$, возможной благодаря симметрии $h(x, y, \alpha)=-h(-x, y,-\alpha)$.

В случаях (22), (23) подходящие растяжения и, если необходимо, инверсии переменных $x_{2}, x_{3}, x_{4}$ позволяют привести $h^{12}, h^{23}, h^{34}$ к виду (20) без свободного члена; таким образом, мы приходим к тому же случаю при $\delta=0$. 
Многочлен (21) отвечает уравнению (31). Это наиболее простой случай, так как параметры фиксируются уже условием (5).

В случае (24) подходящие сдвиги и, если необходимо, смена знака переменных $x_{2}, x_{3}, x_{4}$ позволяют привести $h^{12}, h^{23}, h^{34}$ к виду $2 h(x, y, \alpha)=\alpha^{-1}(x-y)^{2}-\delta \alpha$ при $\delta=1$. Аналогично, в случае (25) подходящее преобразование Мёбиуса общего вида приводит $h^{12}, h^{23}, h^{34}$ к тому же виду с $\delta=0$. В обоих случаях полуинварианты равны $i_{2}=3 \alpha^{-2}, 4 i_{3}=\alpha^{-3}$, следовательно, параметры $h^{12}$ и $h^{34}$ совпадают и дальнейшие замены не нужны. В результате получаем уравнение (32).

\section{§6. Классификация 3D-совместных систем типа $Q$}

Теорема 1 дает очень сильные необходимые условия для 3D-совместности в случае, когда все уравнения относятся к типу $Q$. Это позволит нам классифицировать в этом параграфе все такие системы. На этом заключительном этапе мы должны расположить вокруг куба найденные выше уравнения и выбрать параметры таким образом, чтобы выполнилось условие (14). Это условие может привести к смене знака или инверсии одного из параметров.

В следующей теореме мы возвращаемся к обозначениям переменных и параметров, отвечающим сдвигам на решетке. Порядок уравнений соответствует предыдущей теореме, а обозначения уравнений взяты из [3].

Теорема 4. Любая 3D-совместная система (13) типа $Q$ приводится преобразованиями Мёбиуса к одной из систем из следующего списка:

$$
\begin{aligned}
& \operatorname{sn}\left(\alpha^{(i)}\right) \operatorname{sn}\left(\alpha^{(j)}\right) \operatorname{sn}\left(\alpha^{(i)}-\alpha^{(j)}\right)\left(k^{2} x x_{i} x_{j} x_{i j}+1\right)+\operatorname{sn}\left(\alpha^{(i)}\right)\left(x x_{i}+x_{j} x_{i j}\right) \\
& \quad-\operatorname{sn}\left(\alpha^{(j)}\right)\left(x x_{j}+x_{i} x_{i j}\right)-\operatorname{sn}\left(\alpha^{(i)}-\alpha^{(j)}\right)\left(x x_{i j}+x_{i} x_{j}\right)=0, \\
& \left(\alpha^{(i)}-\frac{1}{\alpha^{(i)}}\right)\left(x x_{i}+x_{j} x_{i j}\right) \\
& \quad-\left(\alpha^{(j)}-\frac{1}{\alpha^{(j)}}\right)\left(x x_{j}+x_{i} x_{i j}\right)-\left(\frac{\alpha^{(i)}}{\alpha^{(j)}}-\frac{\alpha^{(j)}}{\alpha^{(i)}}\right)\left(x x_{i j}+x_{i} x_{j}\right) \\
& \quad-\frac{\delta}{4}\left(\alpha^{(i)}-\frac{1}{\alpha^{(i)}}\right)\left(\alpha^{(j)}-\frac{1}{\alpha^{(j)}}\right)\left(\frac{\alpha^{(i)}}{\alpha^{(j)}}-\frac{\alpha^{(j)}}{\alpha^{(i)}}\right)=0, \\
& \quad+\alpha^{(i)} \alpha^{(j)}\left(\alpha^{(i)}-\alpha^{(j)}\right)\left(x+x_{i}+x_{j}+x_{i j}\right) \\
& \quad-\alpha^{(i)} \alpha^{(j)}\left(\alpha^{(i)}-\alpha^{(j)}\right)\left(\left(\alpha^{(i)}\right)^{2}-\alpha^{(i)} \alpha^{(j)}+\left(\alpha^{(j)}\right)^{2}\right)=0, \\
& \alpha^{(i)}\left(x-x_{j}\right)\left(x_{i}-x_{i j}\right)-\alpha^{(j)}\left(x-x_{i}\right)\left(x_{j}-x_{i j}\right)+\delta \alpha^{(i)} \alpha^{(j)}\left(\alpha^{(i)}-\alpha^{(j)}\right)=0 .
\end{aligned}
$$

Доказательство. Прежде всего, заметим, что уравнения разных типов (29)(32) совместными быть не могут, так как различны соответствующие сингулярные кривые. В частности, параметры $k^{2}$ в случае $(29)$ и $\delta$ в случаях (30), (32) должны быть одни и те же на всех гранях куба. Более того, каждое уравнение списка обладает симметрией квадрата, т. е. инвариантно относительно замен $\left(x_{1} \leftrightarrow x_{2}, x_{3} \leftrightarrow x_{4}\right)$ и $\left(x_{1} \leftrightarrow x_{3}, \alpha \leftrightarrow \beta\right)$.

Таким образом, уравнения на всех гранях могут отличаться лишь значениями $\alpha$ и $\beta$. Рассмотрим уравнения на трех гранях, сходящихся в одной вершине, для 
определенности, $x$ :

$$
Q\left(x, x_{1}, x_{2}, x_{12}, \alpha, \tilde{\beta}\right)=0, \quad Q\left(x, x_{2}, x_{3}, x_{23}, \beta, \tilde{\gamma}\right)=0, \quad Q\left(x, x_{3}, x_{1}, x_{13}, \gamma, \tilde{\alpha}\right)=0 .
$$

Пусть

$$
\delta_{x_{2}, x_{12}} Q\left(x, x_{1}, x_{2}, x_{12}, \alpha, \tilde{\beta}\right)=\kappa(\alpha, \tilde{\beta}) h\left(x, x_{1}, \alpha\right) .
$$

Тогда в силу симметрии

$$
\delta_{x_{1}, x_{12}} Q\left(x, x_{1}, x_{2}, x_{12}, \alpha, \tilde{\beta}\right)=\kappa(\tilde{\beta}, \alpha) h\left(x, x_{2}, \tilde{\beta}\right)
$$

и, согласно теореме 1, параметры должны быть связаны следующим образом:

$$
\begin{gathered}
\frac{h\left(x, x_{1}, \alpha\right)}{h\left(x, x_{1}, \tilde{\alpha}\right)}=m(\alpha, \tilde{\alpha}), \quad \frac{h\left(x, x_{2}, \beta\right)}{h\left(x, x_{2}, \tilde{\beta}\right)}=m(\beta, \tilde{\beta}), \quad \frac{h\left(x, x_{3}, \gamma\right)}{h\left(x, x_{3}, \tilde{\gamma}\right)}=m(\gamma, \tilde{\gamma}), \\
\frac{\kappa(\alpha, \tilde{\beta}) \kappa(\beta, \tilde{\gamma}) \kappa(\gamma, \tilde{\alpha})}{\kappa(\tilde{\beta}, \alpha) \kappa(\tilde{\gamma}, \beta) \kappa(\tilde{\alpha}, \gamma)} m(\alpha, \tilde{\alpha}) m(\beta, \tilde{\beta}) m(\gamma, \tilde{\gamma})=-1 .
\end{gathered}
$$

В случае (29) прямое вычисление показывает, что $\kappa(\alpha, \beta)=2 \operatorname{sn}(\alpha) \operatorname{sn}(\beta) \operatorname{sn}(\alpha+$ $\beta$ и и

$$
h(x, y, \alpha)=\frac{1}{2 \operatorname{sn}(\alpha)}\left(k^{2} \operatorname{sn}^{2}(\alpha) x^{2} y^{2}+2 \operatorname{sn}^{\prime}(\alpha) x y-x^{2}-y^{2}+\operatorname{sn}^{2}(\alpha)\right),
$$

следовательно, $\tilde{\alpha}$ может принимать значения $\pm \alpha$ и аналогично обстоит дело с $\beta, \gamma$. Очевидно, что с точностью до перенумерации, возможны два случая:

$$
\tilde{\alpha}=-\alpha, \quad \tilde{\beta}=-\beta, \quad \tilde{\gamma}=-\gamma \quad \text { или } \quad \tilde{\alpha}=\alpha, \quad \tilde{\beta}=\beta, \quad \tilde{\gamma}=-\gamma .
$$

Более того, это фактически один и тот же случай, так как мы можем сделать замену $(\alpha, \tilde{\beta}) \rightarrow(-\alpha,-\tilde{\beta})$, не меняющую уравнение $Q\left(x, x_{1}, x_{2}, x_{12}, \alpha, \tilde{\beta}\right)=0$, как легко видно из (29). Нетрудно проверить, что расстановка знаков на всем кубе всегда приводится к той, что указана в системе $\left(Q_{4}\right)$.

Далее, рассмотрим случай (30). Здесь

$$
\begin{gathered}
\kappa(\alpha, \beta)=-\frac{\left(1-\alpha^{2} \beta^{2}\right)\left(1-\alpha^{2}\right)\left(1-\beta^{2}\right)}{\alpha^{2} \beta^{2}} \\
h(x, y, \alpha)=\frac{\alpha}{1-\alpha^{2}}\left(x^{2}+y^{2}\right)-\frac{1+\alpha^{2}}{1-\alpha^{2}} x y+\frac{\left(1-\alpha^{2}\right) \delta}{4 \alpha}
\end{gathered}
$$

и $\tilde{\alpha}=\alpha$ или $\tilde{\alpha}=1 / \alpha$. Учитывая инвариантность уравнения (30) относительно одновременной инверсии $\alpha, \beta$, можно положить, не теряя общности,

$$
\tilde{\alpha}=1 / \alpha, \quad \tilde{\beta}=1 / \beta, \quad \tilde{\gamma}=1 / \gamma,
$$

что приводит к системе $\left(Q_{3}\right)$. В случаях $(31),(32)$ имеем соответственно

$$
\begin{gathered}
\kappa(\alpha, \beta)=-4 \alpha \beta(\alpha+\beta), \quad h(x, y, \alpha)=\frac{1}{4 \alpha}(x-y)^{2}-\frac{\alpha}{2}(x+y)+\frac{\alpha^{3}}{4}, \\
\kappa(\alpha, \beta)=-2 \alpha \beta(\alpha+\beta), \quad h(x, y, \alpha)=\frac{1}{2 \alpha}(x-y)^{2}-\frac{\alpha \delta}{2},
\end{gathered}
$$

и можно положить $\tilde{\alpha}=-\alpha, \tilde{\beta}=-\beta, \tilde{\gamma}=-\gamma$ в точности, как и выше. Это дает системы $\left(Q_{2}\right),\left(Q_{1}\right)$. 
Основное уравнение $\left(Q_{4}\right)$ списка впервые было выведено в [2] и далее изучалось в [5]. Представление Лакса для $\left(Q_{4}\right)$ было найдено в [19] методом, основанным на трехмерной совместности. Якобиева форма $\left(Q_{4}\right)$, представленная в теореме 4 , была найдена в [14]. Уравнения $\left(Q_{1}\right)$ и $\left(\left.Q_{3}\right|_{\delta=0}\right)$ восходят к [21]. Уравнения $\left(Q_{2}\right)$ и $\left(\left.Q_{3}\right|_{\delta=1}\right)$ в явном виде появились впервые в [3].

\section{§7. Примеры систем типа $H$}

В отличие от систем типа $Q$, системы типа $H$ можно рассматривать как «вырожденные». Их классификация представляется довольно утомительной задачей, и сейчас мы не можем предложить эффективной процедуры ее решения. С другой стороны, примеры из $\S 3$ показывают, что этот класс нельзя просто отбросить как «патологический». Действительно, пример дискретного КдФ (17) наводит на мысль, что в некоторых случаях вырождение биквадратичной кривой может быть несущественным обстоятельством, не отражающимся на свойствах интегрируемости уравнения. Здесь мы рассмотрим еще несколько примеров такого сорта, отвечающих случаям (22), (23) при $\lambda \mu=0,(24)$ при $\lambda=0$ и (25) при $\varkappa \nu-\lambda \mu=0$, которые мы исключили из рассмотрения в предыдущем параграфе. Оказывается, что если мы применим тот же алгоритм к этим случаям (хотя теперь для этого нет оснований), то будет воспроизведен список $H$ из нашей предыдущей статьи [3]:

$$
\begin{aligned}
& \alpha^{(i)}\left(x x_{i}+x_{j} x_{i j}\right)-\alpha^{(j)}\left(x x_{j}+x_{i} x_{i j}\right)+\delta\left(\left(\alpha^{(i)}\right)^{2}-\left(\alpha^{(j)}\right)^{2}\right)=0, \\
& \left(x-x_{i j}\right)\left(x_{i}-x_{j}\right)+\left(\alpha^{(j)}-\alpha^{(i)}\right)\left(x+x_{i}+x_{j}+x_{i j}\right)+\left(\alpha^{(j)}\right)^{2}-\left(\alpha^{(i)}\right)^{2}=0, \\
& \left(x-x_{i j}\right)\left(x_{i}-x_{j}\right)+\alpha^{(j)}-\alpha^{(i)}=0 .
\end{aligned}
$$

Можно непосредственно проверить, что все утверждения теоремы 1 остаются верны для этих уравнений, хотя биквадратичные кривые и вырожденны.

Рассматривая асимметричные случаи (26), (27), (28) с разными многочленами в вершинах, мы находим, что возможны следующие варианты, с точностью до перестановок (ясно, что когда рассматривается одно уравнение, диагонали и стороны равноправны):

$$
\begin{array}{cc}
\left(x_{1}^{2}-1, x_{2}^{2}, x_{3}^{2}, x_{4}^{2}\right), \quad\left(x_{1}^{2}-1, x_{2}^{2}-1, x_{3}^{2}, x_{4}^{2}\right), & \left(x_{1}^{2}-1, x_{2}^{2}-1, x_{3}^{2}-1, x_{4}^{2}\right), \\
\left(x_{1}, 1,1,1\right), \quad\left(x_{1}, x_{2}, 1,1\right), & \left(x_{1}, x_{2}, x_{3}, 1\right), \\
(1,0,0,0), \quad(1,1,0,0), & (1,1,1,0) .
\end{array}
$$

Прямая проверка показывает, что варианты типа $\left(\begin{array}{ll}r_{2}\left(x_{4}\right) & r_{1}\left(x_{3}\right) \\ r_{1}\left(x_{1}\right) & r_{2}\left(x_{2}\right)\end{array}\right)$ реализуются и приводят к следующему списку $3 \mathrm{D}$-совместных уравнений:

$$
\begin{aligned}
& \alpha\left(x_{1} x_{2}+x_{3} x_{4}\right)-\beta\left(x_{1} x_{4}+x_{2} x_{3}\right)+\left(\alpha^{2}-\beta^{2}\right)\left(\delta+\frac{\varepsilon x_{2} x_{4}}{\alpha \beta}\right)=0, \\
& \left(x_{1}-x_{3}\right)\left(x_{2}-x_{4}\right)+(\beta-\alpha)\left(x_{1}+x_{2}+x_{3}+x_{4}\right)+\beta^{2}-\alpha^{2} \\
& \quad+\varepsilon(\beta-\alpha)\left(2 x_{2}+\alpha+\beta\right)\left(2 x_{4}+\alpha+\beta\right)+\varepsilon(\beta-\alpha)^{3}=0, \\
& \left(x_{1}-x_{3}\right)\left(x_{2}-x_{4}\right)+(\beta-\alpha)\left(1+\varepsilon x_{2} x_{4}\right)=0 .
\end{aligned}
$$


Этот список можно рассматривать как деформацию списка $H$, который отвечает случаю $\varepsilon=0$. Однако здесь мы использовали обозначение с циклическими индексами вместо сдвиговых, так как из-за потери симметрии расположение уравнений на гранях куба требует более явного описания (см. ниже). Отметим, что в $\left(H_{1}^{\varepsilon}\right)$ многочлен $1+\varepsilon x_{2} x_{4}$ можно заменить на многочлен $\kappa x_{2} x_{4}+\mu\left(x_{2}+x_{4}\right)+\nu$ с произвольными коэффициентами. Соответствующие биквадратичные многочлены и их дискриминанты указаны в следующей таблице (с точностью до нормировки $Q \rightarrow \mu(\alpha, \beta) Q)$ :

\begin{tabular}{c|ccc} 
& $h\left(x_{1}, x_{2}\right)$ & $r_{1}\left(x_{1}\right)$ & $r_{2}\left(x_{2}\right)$ \\
\hline$\left(H_{3}^{\varepsilon}\right)$ & $x_{1} x_{2}+\varepsilon \alpha^{-1} x_{2}^{2}+\delta \alpha$ & $x_{1}^{2}-4 \delta \varepsilon$ & $x_{2}^{2}$ \\
$\left(H_{2}^{\varepsilon}\right)$ & $x_{1}+x_{2}+\alpha+2 \varepsilon\left(x_{2}+\alpha\right)^{2}$ & $1-8 \varepsilon x_{1}$ & 1 \\
$\left(H_{1}^{\varepsilon}\right)$ & $1+\varepsilon x_{2}^{2}$ & $-4 \varepsilon$ & 0
\end{tabular}

Каждое из этих уравнений обладает симметрией ромба

$$
Q\left(x_{1}, x_{2}, x_{3}, x_{4}, \alpha, \beta\right)=-Q\left(x_{3}, x_{2}, x_{1}, x_{4}, \beta, \alpha\right)=-Q\left(x_{1}, x_{4}, x_{3}, x_{2}, \beta, \alpha\right),
$$

но не квадрата, так как вершинам $x_{1}, x_{2}$ отвечают многочлены с нулями разной кратности. Уравнения 3D-совместны на черно-белой решетке $i+j+k(\bmod 2)$. То есть каждой грани соответствует копия уравнения, причем так, что параметры на противоположных ребрах совпадают и $x, x_{12}, x_{13}, x_{23}$ - вершины одного типа (здесь мы опять переключаемся на обозначения при помощи сдвигов, как на рис. 3):

$$
\begin{gathered}
Q\left(x, x_{i}, x_{i j}, x_{j}, \alpha^{(i)}, \alpha^{(j)}\right)=0, \quad Q\left(x_{i k}, x_{k}, x_{j k}, x_{123}, \alpha^{(i)}, \alpha^{(j)}\right)=0, \\
\{i, j, k\}=\{1,2,3\} .
\end{gathered}
$$

Очевидно, что уравнения на противоположных гранях куба не совпадают, но тем не менее систему можно распространить на всю решетку $\mathbb{Z}^{3}$. Выполняется свойство тетраэдральности.

Наконец, отметим, что удается также скомбинировать уравнения с симметрией квадрата и трапеции. Рассмотрим опять уравнение $\left(Q_{1}\right)$. Пусть одной паре противоположных граней отвечают уравнения

$$
Q_{1}\left(x, x_{1}, x_{12}, x_{2} ; \alpha^{(1)}, \alpha^{(2)}\right)_{\delta=1}=0, \quad Q_{1}\left(x_{3}, x_{13}, x_{123}, x_{23} ; \alpha^{(1)}, \alpha^{(2)}\right)_{\delta=0}=0,
$$

а двум остальным парам - уравнения

$$
Q\left(x, x_{i}, x_{i, 3}, x_{3}, \alpha^{(i)}, \varepsilon\right)=0, \quad Q\left(x_{j}, x_{i j}, x_{123}, x_{j, 3}, \alpha^{(i)}, \varepsilon\right)=0, \quad\{i, j\}=\{1,2\},
$$

где многочлен

$$
Q\left(x_{1}, x_{2}, x_{3}, x_{4}, \gamma, \varepsilon\right)=\left(x_{1}-x_{2}\right)\left(x_{3}-x_{4}\right)+\gamma\left(\varepsilon^{-1}-\varepsilon x_{3} x_{4}\right)
$$

фактически тот же, что и в $\left(H_{1}^{\varepsilon}\right)$, с точностью до перестановки $x_{2}, x_{3}$. Это неуклюжее сооружение оказывается 3D-совместным и даже обладает свойством тетраэдральности. Эта система также может быть продолжена на решетку $\mathbb{Z}^{3}$. 


\section{§8. Заключительные замечания}

Известны некоммутативные аналоги некоторых уравнений $Q$-типа. В частности, квантовая версия $\left(\left.Q_{1}\right|_{\delta=0}\right)$ появилась в [27]. В [11] подход, основанный на совместности, был сформулирован в некоммутативном контексте, с полями, принимающими значения в произвольной ассоциативной алгебре. Само определение трехмерной совместности в этом случае остается прежним, но предположение о мультиаффинности заменяется на требование, чтобы уравнение можно было привести к линейному виду $p x=q$ относительно любой переменной $x$. Эти два свойства в некоммутативном случае не эквивалентны, как видно из следующих примеров. Первый был найден в [11], а два других найдены В. В. Соколовым и В. Э. Адлером (не опубликовано):

$$
\begin{array}{ll}
\alpha^{(1)}\left(x-x_{2}\right)\left(x_{2}-x_{12}\right)^{-1}=\alpha^{(2)}\left(x-x_{1}\right)\left(x_{1}-x_{12}\right)^{-1}, & \left(\left.\widehat{Q}_{1}\right|_{\delta=0}\right) \\
\alpha^{(1)}\left(x_{1}-x_{12}+\alpha^{(2)}\right)\left(x-x_{1}-\alpha^{(1)}\right)^{-1} & \\
\quad=\alpha^{(2)}\left(x_{2}-x_{12}+\alpha^{(2)}\right)\left(x-x_{2}-\alpha^{(2)}\right)^{-1}, & \left(\left.\widehat{Q}_{1}\right|_{\delta=1}\right) \\
\left(1-\left(\alpha^{(1)}\right)^{2}\right)\left(x_{1}-\alpha^{(2)} x_{12}\right)\left(\alpha^{(1)} x-x_{1}\right)^{-1} & \\
\quad=\left(1-\left(\alpha^{(2)}\right)^{2}\right)\left(x_{2}-\alpha^{(1)} x_{12}\right)\left(\alpha^{(2)} x-x_{2}\right)^{-1} . & \left(\left.\widehat{Q}_{3}\right|_{\delta=0}\right)
\end{array}
$$

Существование некоммутативных аналогов уравнений $\left(Q_{2}\right),\left(\left.Q_{3}\right|_{\delta=1}\right)$ и $\left(Q_{4}\right)$ остается открытым вопросом. Хотя анализ сингулярных решений может быть важен и в этой задаче как общий принцип, наша техника, основанная на алгебраических свойствах мультиаффинных и биквадратичных многочленов, непосредственно не применима. Более общие квантовые системы со свойством совместности найдены недавно в [8], [7].

\section{ЛитеРАТУРА}

[1] В. И. Арнольд, Математические методы классической механики, Наука, М., 1974.

[2] V. E. Adler, Bäcklund transformation for the Krichever-Novikov equation, Internat. Math. Res. Notices, 1 (1998), 1-4.

[3] V. E. Adler, A. I. Bobenko, Yu. B. Suris, Classification of integrable equations on quad-graphs. The consistency approach, Comm. Math. Phys., 233:3 (2003), 513-543.

[4] V. E. Adler, A. I. Bobenko, Yu. B. Suris, Geometry of Yang-Baxter maps: pencils of conics and quadrirational mappings, Comm. Anal. Geom., 12:5 (2004), 967-1007.

[5] V. E. Adler, Yu. B. Suris, Q4: Integrable master equation related to an elliptic curve, Internat. Math. Res. Notices, 47 (2004), 2523-2553.

[6] V. E. Adler, A. P. Veselov, Cauchy problem for integrable discrete equations on quadgraphs, Acta Appl. Math., 84:2 (2004), 237-262.

[7] V. Bazhanov, V. Mangazeev, S. Sergeev, Faddeev-Volkov solution of the Yang-Baxter equation and discrete conformal symmetry, Nuclear Phys. B, 784:3 (2007), 234-258.

[8] V. Bazhanov, S. Sergeev, Zamolodchikov's tetrahedron equation and hidden structure of quantum groups, J. Phys. A., 39:13 (2006), 3295-3310.

[9] L. Bianchi, Vorlesungen über Differenzialgeometrie, Teubner, Leipzig, 1899.

[10] A. I. Bobenko, Yu. B. Suris, Integrable systems on quad-graphs, Internat. Math. Res. Notices, 11 (2002), 573-611.

[11] A. I. Bobenko, Yu. B. Suris, Integrable non-commutative equations on quad-graphs. The consistency approach, Lett. Math. Phys., 61:3 (2002), 241-254. 
[12] A. I. Bobenko, Yu. B. Suris, Discrete Differential Geometry. Integrable Structure, Graduate Studies in Math., vol. 98, Amer. Math. Soc., Providence, RI, 2008.

[13] J. Hietarinta, A new two-dimensional lattice model that is "consistent around a cube", J. Phys. A, 37:6 (2004), L67-L73.

[14] J. Hietarinta, Searching for CAC-maps, J. Nonlinear Math. Phys., 12, Suppl. 2 (2005), $223-230$.

[15] Р. М. Кашаев, И. Г. Корепанов, С. М. Сергеев, Функииональное уравнение тетраэдров, ТМФ, 117:3 (1998), 370-384.

[16] I. G. Korepanov, Algebraic Integrable Dynamical Systems, 2+1-Dimensional Models in Wholly Discrete Space-Time, and Inhomogeneous Models in 2-Dimensional Statistical Physics, http://arxiv.org/abs/solv-int/9506003.

[17] J.-M. Maillet, F. W. Nijhoff, Integrability for multidimensional lattice models, Phys. Lett. B, 224:4 (1989), 389-396.

[18] А. В. Михайлов, А. Б. Шабат, Р. И. Ямилов, Симметрийный подход к классификации нелинейных уравнений. Полные списки интегрируемых систем, УМН, 42:4 (1987), 3-53.

[19] F. W. Nijhoff, Lax pair for the Adler (lattice Krichever-Novikov) system, Phys. Lett. A, 297 (2002), 49-58.

[20] V. Papageorgiou, A. Tongas, A. Veselov, Yang-Baxter maps and symmetries of integrable equations on quad-graphs, J. Math. Phys, 47:8 (2006), 083502.

[21] G. R. W. Quispel, F. W. Nijhoff, H. W. Capel, J. van der Linden, Linear integral equations and nonlinear difference-difference equations, Phys. A, 125:2-3 (1984), 344380.

[22] A. Ramani, N. Joshi, B. Grammaticos, T. Tamizhmani, Deconstructing an integrable lattice equation, J. Phys. A, 39:8 (2006), L145-L149.

[23] Yu. B. Suris, A. P. Veselov, Lax pairs for Yang-Baxter maps, J. Nonlinear Math. Phys., 10:suppl. 2 (2003), 223-230.

[24] А. П. Веселов, Интегрируемые отображения, УМН, 46:5 (1991), 3-45.

[25] A. P. Veselov, Yang-Baxter maps: dynamical point of view, in: Combinatorial Aspect of Integrable Systems, MSJ Mem., vol. 17, Math. Soc. Japan, Tokyo, 2007, 145-167.

[26] C. Viallet, Algebraic Entropy for Lattice Equations, http://arxiv.org/abs/math-ph/ 0609043.

[27] A. Volkov, Quantum lattice KdV equation, Lett. Math. Phys., 39:4 (1997), 313-329.

[28] E. T. Whittaker, G. N. Watson, A course of modern analysis, Cambridge Univ. Press, 1927, reprinted in 1996.

Институт теоретической физики им. Л. Д. Ландау РАН e-mail: adler@itp.ac.ru

Institut für Mathematik, Technische Universität Berlin 4 июня 2007 г.

e-mail: bobenko@math.tu-berlin.de

Zentrum Mathematik, Technische Universität München

e-mail: suris@ma.tum.de 